\title{
Multiwavelength/Brillouin Generation in Bismuth-doped Fiber Laser with Single- and Double-Frequency Spacing
}

\author{
Harith Ahmad, Muhamad Z. Samion, Aizuddin A. Kamely, Siyi Wang, Yu Wang, and Jayanta K.
} Sahu

\begin{abstract}
Multiwavelength fiber lasers operating in the $1.3 \mu \mathrm{m}$ wavelength region are of great interest as they complement existing systems in the conventional optical communication band of $1.55 \mu \mathrm{m}$. In this work, a multiwavelength Brillouin fiber laser with single- and double-Brillouin frequency spacings operating in 1.3 $\mu \mathrm{m}$ is proposed and demonstrated using a dispersion compensating fiber (DCF) as the Brillouin gain medium. A bismuth-doped fiber serves as the laser gain medium. At the maximum pump power of $1021 \mathrm{~mW}$ and with the Brillouin pump set at $11 \mathrm{dBm}$, up to 13 Brillouin Stokes lines with a wavelength spacing of $0.074 \mathrm{~nm}$ are obtained in the single-spaced configuration. In the case of the double-spaced configuration, 20 Stokes lines are generated with a wavelength spacing of $0.15 \mathrm{~nm}$ between the even Stokes lines. By tuning the central wavelength of the Brillouin pump, both laser configurations are wavelengthtunable with a range of $93 \mathrm{~nm}$ and $19 \mathrm{~nm}$ for the single-spaced and double-spaced Brillouin lasers respectively. This is, to the best of the author's knowledge, the first demonstration of a multiwavelength/Brillouin laser using a bismuth-doped fiber as the laser gain medium.
\end{abstract}

Index Terms - Bismuth-doped fiber laser; multiwavelength; stimulated Brillouin Scattering; single-frequency spacing, doublefrequency spacing.

\section{INTRODUCTION}

$\mathrm{T}$ he advent of multiwavelength laser sources has prompted a revolution in telecommunication [1], [2], sensing applications [3], [4], optical signal processing and microwave photonics technology [5], [6]. Among them, multiwavelength fiber laser sources have attracted substantial attention ascribed to their flexible wavelength tuning capabilities [7], [8], high signal-to-noise ratios (SNRs) [9], small power fluctuation as well as long operational lifetimes [10]. Additionally, their ease of fabrication has yielded various inventive designs and approaches to realize multiwavelength operation in fiber laser systems. These include the use of optical nonlinear

Manuscript received month day, year; revised month day, year; accepted month day, year. This work was supported in part by the Ministry of Higher Education, Malaysia under the Grants LRGS (2015) NGOD/UM./KPT and in part by the University of Malaya under the Grant RU 010-2016. (Corresponding author: Harith Ahmad.)

Harith Ahmad is with the Photonics Research Centre, University of Malaya, Kuala Lumpur 50603, Malaysia and the Department of Physics, Faculty of Science, University of Malaya, Kuala Lumpur 50603, Malaysia (e-mail: harith@um.edu.my) mirrors [11], fiber Bragg gratings (FBGs) [12], Mach-Zehnder interferometers (MZIs) [13], and also the exploitation of nonlinear effects such as four-wave mixing (FWM) [14], and stimulated Brillouin scattering (SBS) [15]. Among these, the use of the SBS effect in optical fibers is particularly interesting for multiwavelength generation as the lasers would exhibit extraordinarily narrow linewidth, low intensity noise and a stable operation at room temperature [16]-[18]. This was favored for applications in the dense-multiwavelength-division multiplexed (DWDM) systems.

To date, a number of studies have developed the SBS based multiwavelength fiber lasers with improving performances in terms of number of channels, frequency spacing, operating wavelength and Brillouin Stokes tuning capability. For instance, in 2011, Tang et. al [19] demonstrated a multiwavelength Brillouin-erbium fiber laser in a simple ring cavity configuration with an operating wavelength range from 1564 to $1576 \mathrm{~nm}$. Later, Alimi et. al [20] successfully demonstrated a multiwavelength Brillouin-erbium fiber laser in a linear cavity structure with a wide Brillouin Stokes lines tuning capabilities, covering from 1532 to $1572 \mathrm{~nm}$. Meanwhile, Wang et al [21] reported an SBS-based multiwavelength generation in $2.0 \mu \mathrm{m}$ region with the thuliumdoped fiber laser, obtaining a wavelength spacing of $0.10 \mathrm{~nm}$. Soon after, $\mathrm{Hu}$ et. al [22] reported a switchable single and double Brillouin frequency spacing multiwavelength fiber laser based on a dual scheme consisting of a Brillouin gain medium and a thulium-doped fiber. A wavelength tunability of $1.3 \mathrm{~nm}$ is also realized by shifting the Brillouin pump wavelength. Recently, Ahmad et al [23] successfully demonstrated a multiwavelength Brillouin fiber laser operating in the $1.3 \mu \mathrm{m}$ region, utilizing a praseodymium-doped fluoride fiber as the amplifying gain medium. As for now, this was the only reported work on the generation of multiwavelength Brillouin output in the $1.3 \mu \mathrm{m}$ region using a rare earth-doped fiber as the active

Muhamad Z. Samion and Aizuddin A. Kamely are with the Photonics Research Centre, University of Malaya, Kuala Lumpur 50603, Malaysia (e-mail: zarifzf@um.edu.my; aizdeen94@gmail.com).

Siyi Wang, Yu Wang, and Jayanta K. Sahu are with the Optoelectronics Research Centre, University of Southampton, Highfield, Southampton SO17 1BJ, UK (e-mail: siyi.wang@soton.ac.uk; yw9n17@soton.ac.uk; jks@orc.soton.ac.uk). 

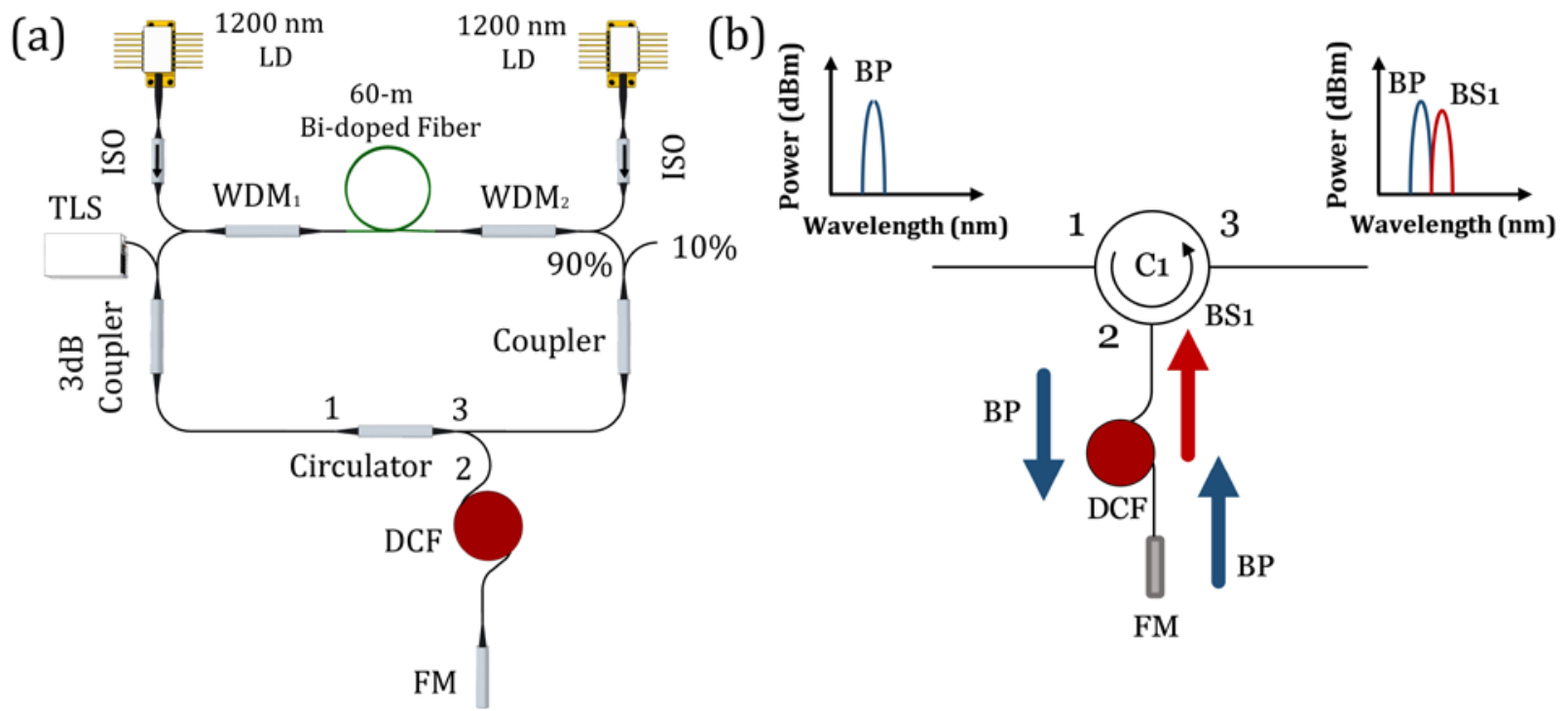

Fig. 1. (a) Experimental setup for single-spacing Brillouin/bismuth-doped fiber laser, (b) Principle of operation for the generation of the single-spaced Brillouin Stokes.

medium. The development of multiwavelength Brillouin fiber lasers in the $1.3 \mu \mathrm{m}$, which is known as the zero-dispersion wavelength region, is of significant interest to extend the capacity of current communication networks. Recently, bismuth-doped phosphosilicate active fibers have shown prominent progress in developing amplifiers and lasers operating in the $1.3 \mu \mathrm{m}$ region [24]-[27]. Furthermore, phosposilicate-based active fibers can be easily spliced with standard single mode fibers (SMFs) with minimal loss as compared to that of fluoride-based active fibers.

In this paper, a multiwavelength/Brillouin Bi-doped fiber laser (MWBBFL) is proposed and demonstrated. A 60-meter bismuth-doped fiber (BiDF) is used as the laser gain medium while a 10-km dispersion compensating fiber (DCF) serves as the Brillouin gain medium (BGM). By changing the placement of the DCF and the configuration of the laser cavity, stable Brillouin Stokes with single- and double-Brillouin frequency spacings are generated in the $1.3 \mu \mathrm{m}$ region. A maximum of 13 single-spaced Stokes lines are obtained with a spacing of $0.074 \mathrm{~nm}$ or $\sim 12.7 \mathrm{GHz}$, while 20 Stokes lines are obtained in the double-spaced Brillouin configuration. The spacing between adjacent double-spaced Stokes are measured to be $0.15 \mathrm{~nm}$, which was in agreement with the calculated frequency spacing of $\sim 25.3 \mathrm{GHz}$. By tuning the Brillouin pump wavelength, the MWBBFL can be broadly tuned over $93 \mathrm{~nm}$ and $19 \mathrm{~nm}$ in the single-spaced and double-spaced wavelength configurations, respectively. This makes the laser as a promising $1.3 \mu \mathrm{m}$ light source that can find application in dense wavelength division multiplexing (DWDM) systems, catering the exponential bandwidth growth in the conventional optical communication band. This is to the best of the author's knowledge the first demonstration of a multi-wavelength Brillouin output using bismuth as the gain medium.

\section{EXPERIMENTAL SETUP \& RESULTS}

A. Single Frequency spacing in Brillouin/Bismuth doped Fiber Laser

The experimental setup for the single-spacing MWBBFL is shown in Fig. 1 (a). The system comprises of two $1200 \mathrm{~nm}$ laser diodes (LDs) that serve as the pump sources for the gain medium, each having a maximum output power of approximately $500 \pm 25 \mathrm{~mW}$. Each $\mathrm{LD}$ is connected to a $1200 \mathrm{~nm}$ isolator (ISO) as a precautionary measure to prevent any signal from damaging the LD. Two 1200/1300 nm wavelength division multiplexers (WDMs) are used to multiplex the pump light into the laser cavity, where the $1200 \mathrm{~nm}$ ports of the WDMs are connected to the output ports of the $1200 \mathrm{~nm}$ isolators. The common ports of both WDMs are directly spliced to a 60-metre BiDF. The bismuth phosphosilicate preform is fabricated by the modified chemical vapor deposition (MCVD), with the combination of the standard solution doping technique. The inner wall of a silica tube is first used to deposit the phosphosilicate soot, and the bismuth dopants are merged together with the soot by soaking the tube in a bismuth precursor solution. The soot is dried and sintered, and finally the tube is collapsed into a bismuth-doped phosphosilicate preform. The preform is finally drawn to form the BiDF that has core and cladding diameters of $12.5 \mu \mathrm{m}$ and $125 \mu \mathrm{m}$. This fibre was fabricated at the Optoelectronic Research Center, University of Southampton, United Kingdom. A Santec WSL-550 tunable laser source (TLS), with a wavelength that is tunable across $1260 \mathrm{~nm}-1365 \mathrm{~nm}$, is used as the Brillouin pump (BP). The BP has a maximum output power of $11 \mathrm{dBm}$ and is injected into the laser cavity through the $50 \%$ port of a $2 \times 13-d B$ coupler. As the TLS has already a built-in isolator, it is directly connected to the coupler. The Brillouin gain medium, which is an approximately $10-\mathrm{km}$ dispersion compensating fiber (DCF), is placed after the Port 2 of a circulator and connected to a Faraday mirror (FM) to reflect the signals back into the laser cavity. The DCF has an affective 
mode area of $55 \mu \mathrm{m}^{2}$ and a dispersion slope of about $0.045 \mathrm{ps} \mathrm{nm}^{-2} \mathrm{~km}^{-1}$ at $1550 \mathrm{~nm}$. A $90 / 10$ coupler is then connected to the Port 3 of the circulator, where $90 \%$ of the signal will circulate back into the cavity while $10 \%$ of the signal is used for optical analysis. A Yokogawa AQ6370C optical spectrum analyzer (OSA) with a resolution of $0.02 \mathrm{~nm}$ is used to record the optical spectrum of the signal.

Fig. 1 (b) shows the operation principle to generate a singlespacing Brillouin Stokes in the proposed setup. As the BP signal enters the ring cavity through the $50 \%$ port of the coupler, it will then propagate towards the DCF by propagating from Port 1 to Port 2 of the circulator. The interaction of the BP signal with the BGM, which in this case the 10-km DCF, will generate the $1^{\text {st }}$ Brillouin stoke $\left(\mathrm{BS}_{1}\right)$ since the $\mathrm{BP}$ at this point exceeds the SBS threshold. $\mathrm{BS}_{1}$ will then travel in the opposite direction as opposed to the $\mathrm{BP}$, which will continue to propagate in the forward direction towards the FM. Both $\mathrm{BS}_{1}$ and the $\mathrm{BP}$ will eventually propagate in the laser cavity through Port 3 and towards the 90/10 coupler, where the multiwavelength output can be observed at the $10 \%$ port of the coupler. As $\mathrm{BS}_{1}$ continues to circulate in the cavity through the $90 \%$ port of the coupler, the $2^{\text {nd }}$ Brillouin stokes $\left(\mathrm{BS}_{2}\right)$ will be generated when $\mathrm{BS}_{1}$ propagates in the DCF. This process will be repeated and will continue to produce subsequent Stokes where $\mathrm{BS}_{n,(\mathrm{n}=2,3,4, \ldots)}$ through the cascaded SBS process as long as the Stokes power exceed the SBS threshold. The Brillouin Stokes will be generated at a certain frequency downshift from its preceding signal, which is the $\mathrm{BP}$ for $\mathrm{BS}_{1}$ and $\mathrm{BS}_{n-1}$ for $\mathrm{BS}_{n}$. The Brillouin frequency shift, $v_{\mathrm{B}}$ can be calculated by the following equation as in [28],

$$
v_{B}=2 n v_{a} / \lambda
$$

where $n$ is the refractive index at the operating wavelength, $v_{\mathrm{a}}$ is the acoustic velocity and $\lambda$ is the operating wavelength of the signal. Taking values that are applicable for silica fibers [29], $\mathrm{n}=1.44$ and $v_{\mathrm{a}}=5.83 \mathrm{~km} / \mathrm{s}, v_{\mathrm{b}}$ is calculated to be $\sim 12.77 \mathrm{GHz}$ at $\lambda=1314 \mathrm{~nm}$.

Fig. 2 shows the evolution of the optical spectrum when the power of the $1200 \mathrm{~nm}$ pump laser diodes was increased. The BP is set at a center wavelength of $1313.998 \mathrm{~nm}$ with an output power of $11 \mathrm{dBm}$. This wavelength is chosen since it coincides closely with the peak of the gain curve of this laser cavity. As can be seen from the figure, the number of Stokes generated is dependent on the pump power. Without the pump from the $1200 \mathrm{~nm}$ laser diodes, one Brillouin Stokes can be observed at $1314.072 \mathrm{~nm}$, implying that the power of the BP itself is already sufficient and has exceeded the SBS threshold of the BGM to generate $\mathrm{BS}_{1}$. The average wavelength spacing between the adjacent Stokes lines was measured to be $0.074 \mathrm{~nm}$, which tallies to the theoretical frequency shift of $\sim 12.77 \mathrm{GHz}$ at about $1314 \mathrm{~nm}$. When the pump power was increased to $362 \mathrm{~mW}$, the $2^{\text {nd }}$ Brillouin Stokes could be observed since $\mathrm{BS}_{1}$ has now been amplified and has enough power to generate $\mathrm{BS}_{2}$. As the pump power is further increased to $482 \mathrm{~mW}, 3$ Stokes lines $\left(\mathrm{BS}_{1}, \mathrm{BS}_{2}\right.$ and $\mathrm{BS}_{3}$ ), and 1 anti-Stokes line were now observed. The generation of anti-Stokes waves can usually be attributed to the interaction between the co-propagating BP and the Stokes waves in the laser cavity, through the FWM effect [30]. More Stokes and anti-Stokes lines are generated as the pump power increases, and the maximum number of Stokes could be observed when the pump power reaches its maximum total value at $1021 \mathrm{~mW}$.

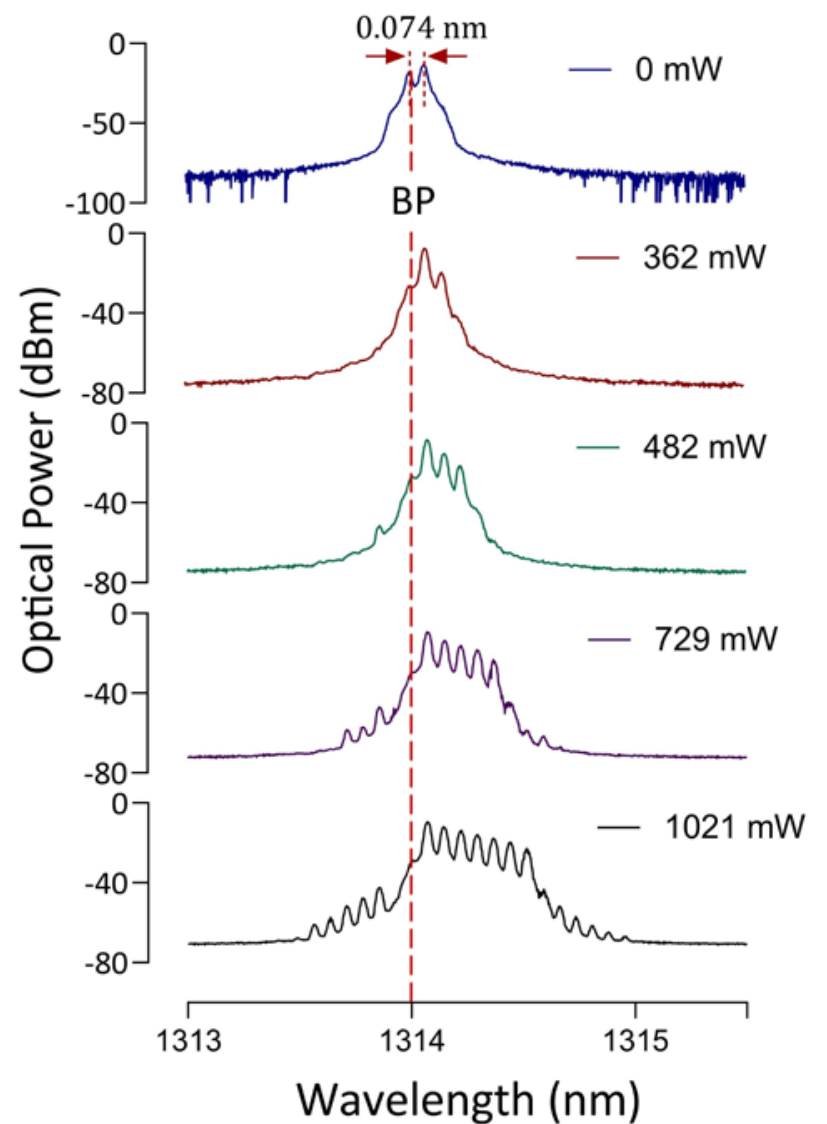

Fig. 2. Evolution of the generated single-spaced Brillouin Stokes with increasing $1200 \mathrm{~nm}$ pump laser diode powers.

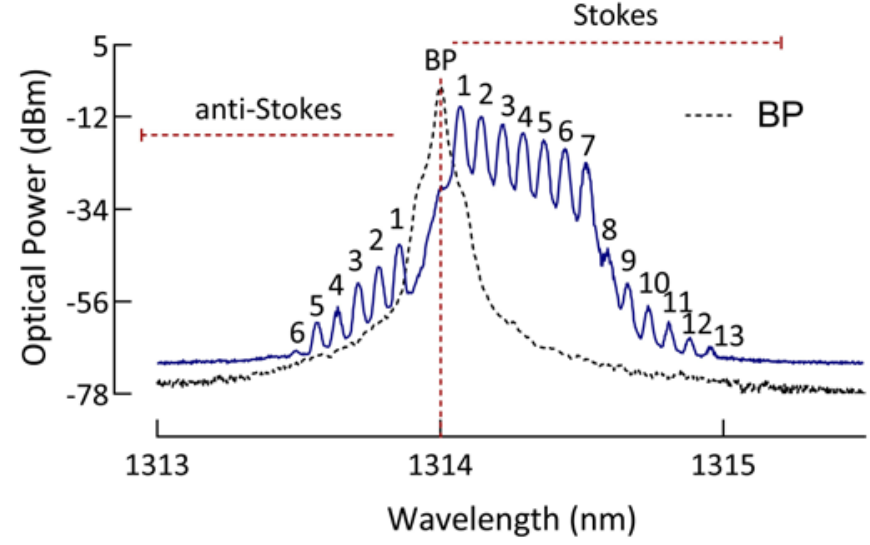

Fig. 3. Optical spectrum of the single-spacing Brillouin frequency at the maximum pump power of $1021 \mathrm{~mW}$.

The output spectrum of the MWBBFL with the maximum number of generated Brillouin stokes is given in Fig. 3. At the maximum pump power of $1021 \mathrm{~mW}$, and without the BP being injected into the cavity, only the amplified spontaneous emission (ASE) was obtained at the output without any lasing. When the BP was launched into the cavity, a total of 13 Brillouin Stokes and 6 anti-Stokes were generated. The 
separation between each anti-Stokes lines was also $0.074 \mathrm{~nm}$, similar to the separation between the Brillouin Stokes. Only the first 7 Stokes generated have SNR values of more than $47 \mathrm{~dB}$, which was calculated from the peak of each Stokes to the noise floor at approximately $-70 \mathrm{dBm}$. The SNR was determined as such because each Stokes has actually its own sharp, distinctive lines when recorded using a high resolution OSA of $0.16 \mathrm{pm}$ as reported in [31]. However, we are unable to obtain the highresolution spectrum at $1.3 \mu \mathrm{m}$ as we do not have a suitable equipment to carry out the measurement at this wavelength region. From the figure, it can be seen that the BP is not as evident as compared to the other Stokes. This could be attributed to the pump depletion after which the Brillouin gain begins to saturate [28].

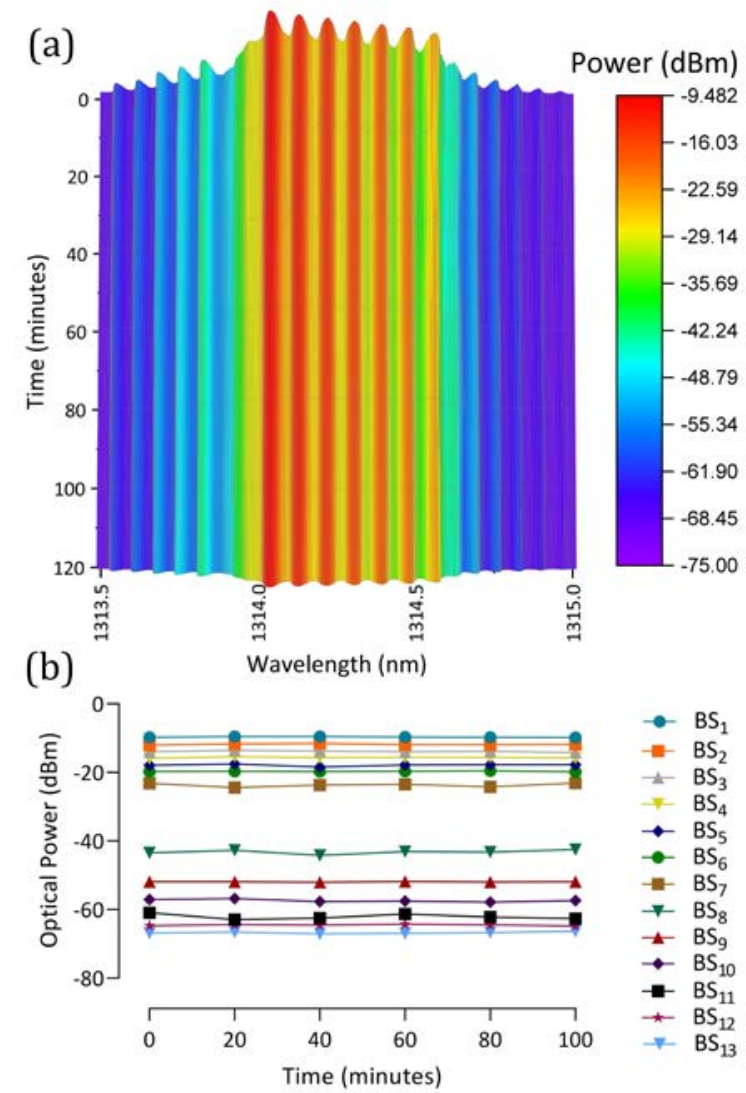

Fig. 4. Stability measurement of the multiwavelength Brillouin fiber laser in terms of the (a) optical spectrum, and (b) the peak power of each single-spaced Brillouin Stokes.

The stability measurement for the single-frequency spacing MWBBFL is recorded and depicted in Fig. 4, with the pump power being fixed at $1021 \mathrm{~mW}$. The output spectrum is taken at every 20-minute interval for a period of 100 minutes. Over the whole period, the MWBBFL shown in Fig. 4 (a) is stable with no major signs of wavelength shifts and power fluctuations. Fig. 4 (b), meanwhile, shows a more detailed analysis of the optical power stability of each Stokes lines. From the graph, the maximum power fluctuations recorded are for $\mathrm{BS}_{8}$ and $\mathrm{BS}_{11}$, which is about $1.7 \mathrm{~dB}$ and $1.9 \mathrm{~dB}$ respectively. Other Stokes lines meanwhile, recorded lower fluctuations of only between $0.25 \mathrm{~dB}$ to $1.37 \mathrm{~dB}$. This implies that the MWBBFL could operate stably for a long period of time.
The tunability of the single-spaced MWBBFL was also investigated by tuning the center wavelength of the $\mathrm{BP}$, where the results are depicted in Fig. 5 (a). The tuning range of the single-spaced MWBBFL covers a broad wavelength span of $93 \mathrm{~nm}$, ranging from $1272 \mathrm{~nm}$ to $1365 \mathrm{~nm}$. Although, it is worthy to note that when the BP is set from $1260 \mathrm{~nm}$ to $1271 \mathrm{~nm}$, only $\mathrm{BS}_{1}$ is observed regardless whether the pump power is at its maximum or even at zero. As mentioned earlier, this is because the power of the BP itself is already sufficient to induce the generation of $\mathrm{BS}_{1}$. The power of $\mathrm{BS}_{1}$ however, does not exceed the SBS threshold and the BiDF could not provide enough amplification for $\mathrm{BS}_{1}$ to generate $\mathrm{BS}_{2}$. Therefore, the tuning range that is considered in this case was when there is more than one Brillouin Stokes being generated.
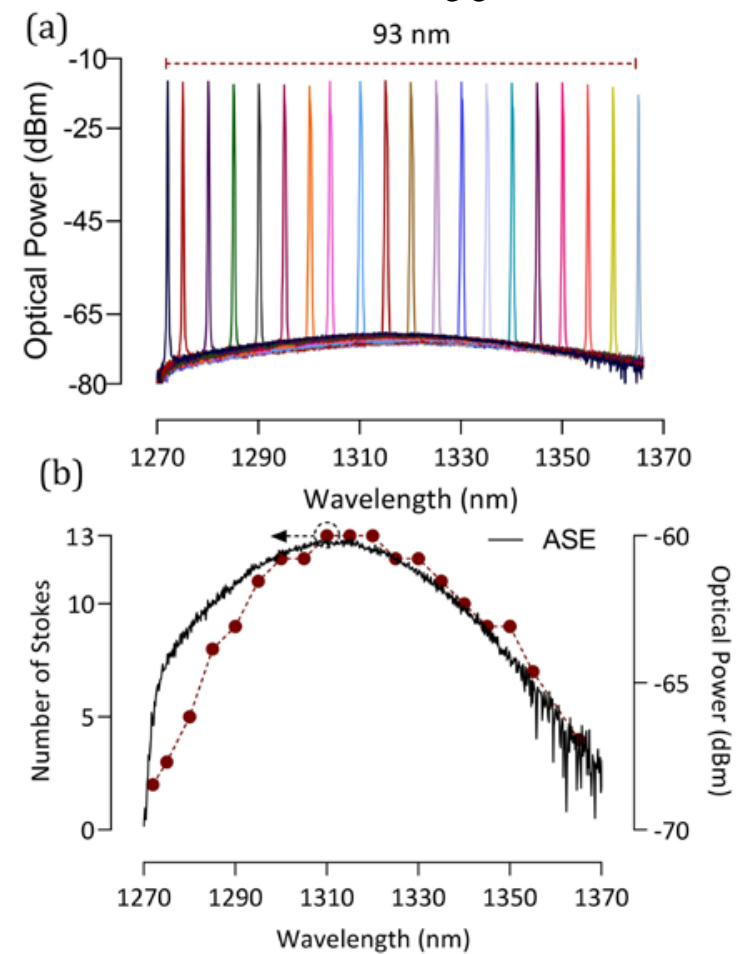

Fig. 5. (a) Tunability spectra of the single-spaced multiwavelength Brillouin fiber laser, and (b) number of generated Brillouin Stokes lines against the BP wavelength.

The number of Brillouin Stokes lines generated at each BP wavelength when the pump power was fixed at $1021 \mathrm{~mW}$ is given in Fig. 5(b). At $1272 \mathrm{~nm}$, there only 2 Stokes lines generated, implying that the BiDF was able to provide enough amplification for $\mathrm{BS}_{1}$ to generate $\mathrm{BS}_{2}$. As the wavelength was tuned towards the longer wavelength, the number of Stokes lines increased gradually until the maximum of 13 Stokes lines were obtained between $1310 \mathrm{~nm}$ to $1320 \mathrm{~nm}$. This is where the peak of the gain curve is located. Tuning the BP wavelength beyond $1320 \mathrm{~nm}$ causes the number of Stokes to decrease until the longest possible wavelength of $1365 \mathrm{~nm}$ is reached, which produced only 4 Stokes. It can be inferred that the trend for the number of Stokes lines generated matches the amplified spontaneous emission (ASE) curve of the BiDF. This indicates that the amplification provided by the BiDF greatly influence the cascading SBS effect for the generation of multiple Stokes lines. 

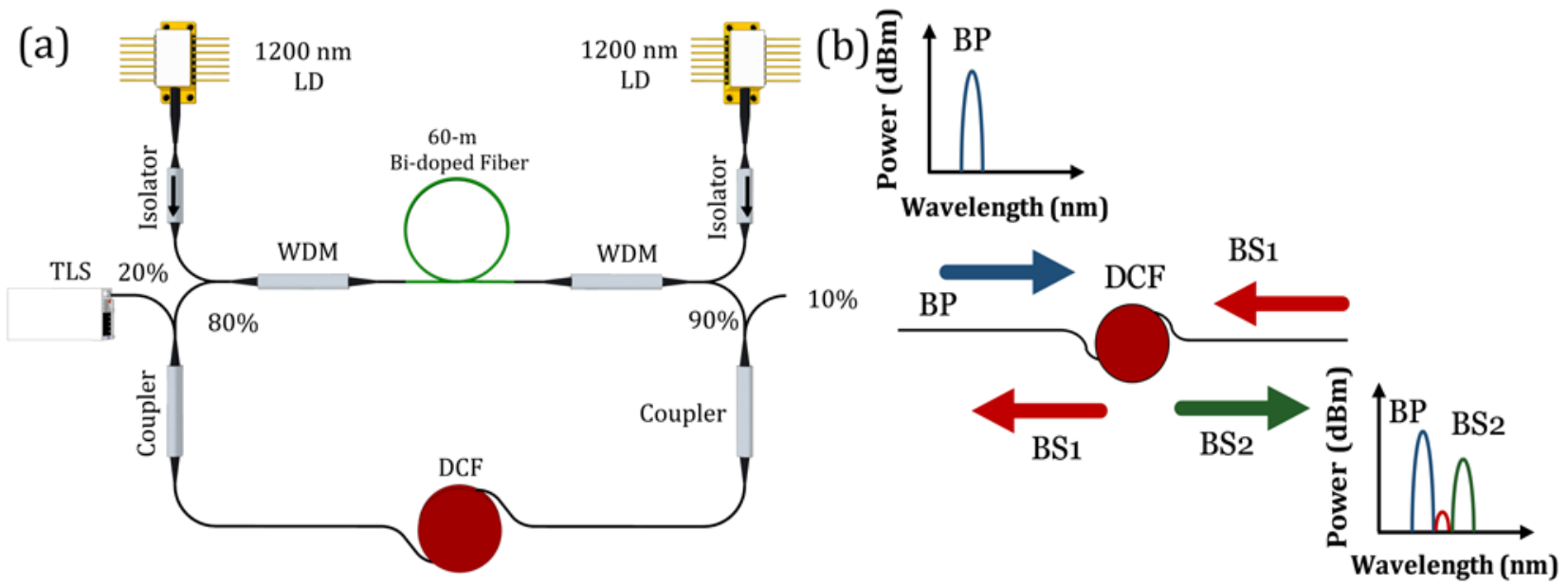

Fig. 6. (a) Experimental setup for the double-spacing Brillouin/bismuth-doped fiber laser, (b) principle of operation for the generation of the double-spaced Brillouin stokes

\section{B. Double Frequency spacing in Brillouin/Bismuth doped Fiber Laser}

The experimental configuration is now modified to generate a double-spaced Brillouin output as given in Fig. 6 (a). The setup mainly comprises of the same components, that was the $1200 \mathrm{~nm}$ LDs, two $1200 \mathrm{~nm}$ ISOs, two 1200/1310 nm WDMs and a $60-\mathrm{m}$ long of $\mathrm{BiDF}$ as the gain medium. No isolator is used in the main cavity for this proposed setup, as to allow a bidirectional signal propagation. For this setup, the BP was injected into the ring cavity through the $20 \%$ port of an $80 / 20$ coupler. This coupler was chosen instead of a 3-dB coupler as to allow a higher circulating signal in the laser cavity. The DCF was placed between the $80 / 20$ and $90 / 10$ couplers. The output signal would be extracted at the $10 \%$ port of the $90 / 10$ coupler.

From Fig. 6 (b), 20\% of the BP signal will be injected into the cavity through the $80 / 20$ coupler and it will travel towards the DCF. The interaction of the BP signal and the DCF would cause a frequency downshift of the oscillating signal thereby generating the $1^{\text {st }}$ Stokes line, $\mathrm{BS}_{1}$ provided the $\mathrm{BP}$ power exceeded the SBS threshold. $\mathrm{BS}_{1}$ will propagate in the backward direction as opposed to the BP, whereas the BP will continue to travel towards the 90/10 coupler and would be extracted at the $10 \%$ output port. When $\mathrm{BS}_{1}$ oscillates in the clockwise direction in the ring cavity, it would be amplified by the BiDF and continue to oscillate towards the other end of the DCF. BS $_{1}$ now acts as the new $\mathrm{BP}$ and can generate $\mathrm{BS}_{2}$ if its power also exceeded the SBS threshold. The generated Stokes line, $\mathrm{BS}_{2}$ will have a wavelength upshift of about $0.074 \mathrm{~nm}$ with respect to $\mathrm{BS}_{1}$ and will travel in the anti-clockwise direction. This process will continue to generate additional Stokes lines as long as each new Stokes line can exceed the SBS threshold. This cavity setup allows the anti-clockwise propagating Stokes lines to be extracted at the $10 \%$ port of the $90 / 10$ coupler. The signals propagating in the clockwise direction will be heavily attenuated as compared to the signals propagating in the anticlockwise direction. This makes the BP and even Stokes to be clearly defined in the extracted optical spectrum at the output, while the odd Stokes were significantly suppressed.

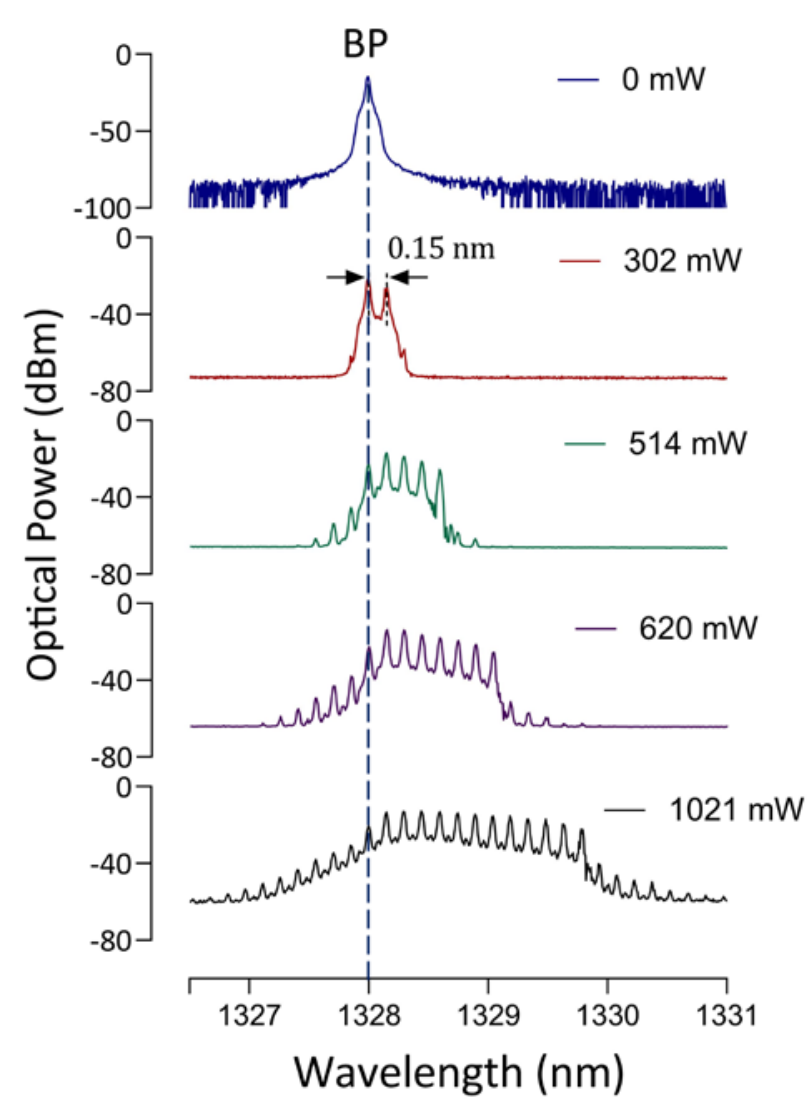

Fig. 7. The evolution of the generated double-spaced Brillouin Stokes lines against the increasing $1200 \mathrm{~nm}$ laser pump power.

The obtained optical spectrum against the increasing $1200 \mathrm{~nm}$ pump power was given in Fig. 7. For the case of the double-spaced MWBBFL, the BP output power was maintained at $11 \mathrm{dBm}$, with the center wavelength being set at $1328 \mathrm{~nm}$ since it is around the peak of the gain curve for this configuration. Similar to the single-spaced MWBBFL, the number of Stokes lines generated is also dependent on the pump power of the pump laser. When both the $1200 \mathrm{~nm}$ pump lasers 
are off, only the BP is observed at the wavelength of 1327.99. $\mathrm{nm}$. As the pump power was increased to $302 \mathrm{~mW}$, $\mathrm{BS}_{2}$ was observed at $1328.14 \mathrm{~nm}$ with a spacing of $0.15 \mathrm{~nm}$ with respect to the BP. This augurs well with the calculated double frequency spacing, which is $\sim 25.3 \mathrm{GHz}$ at around $1328 \mathrm{~nm}$. As the pump power was further increased, more Stokes lines were generated since higher amplification was provided to cascade the SBS effect in the BGM. The pump power was then further increased until a maximum power of $1021 \mathrm{~mW}$, thereby producing the maximum number of doublespaced Brillouin Stokes and anti-Stokes lines.

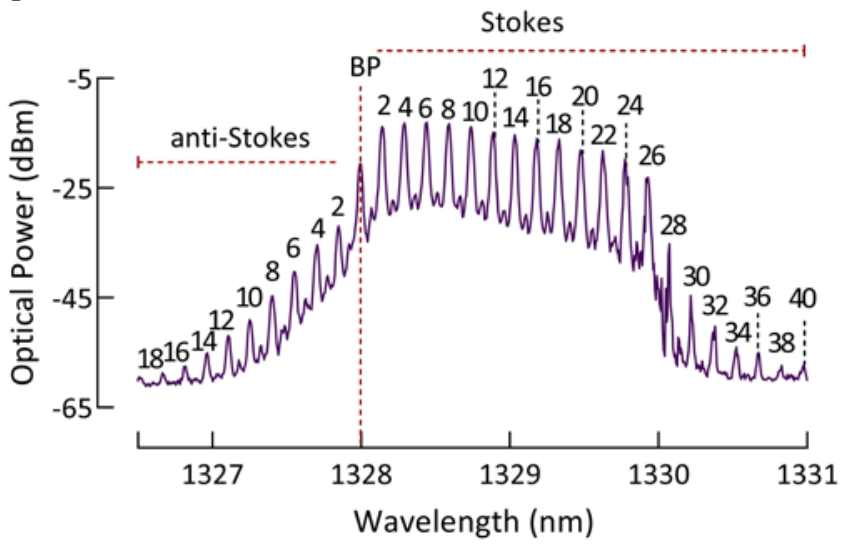

Fig. 8. Optical spectrum of the double-spacing Brillouin Stokes and anti-Stokes lines at the maximum pump power of $1021 \mathrm{~mW}$.
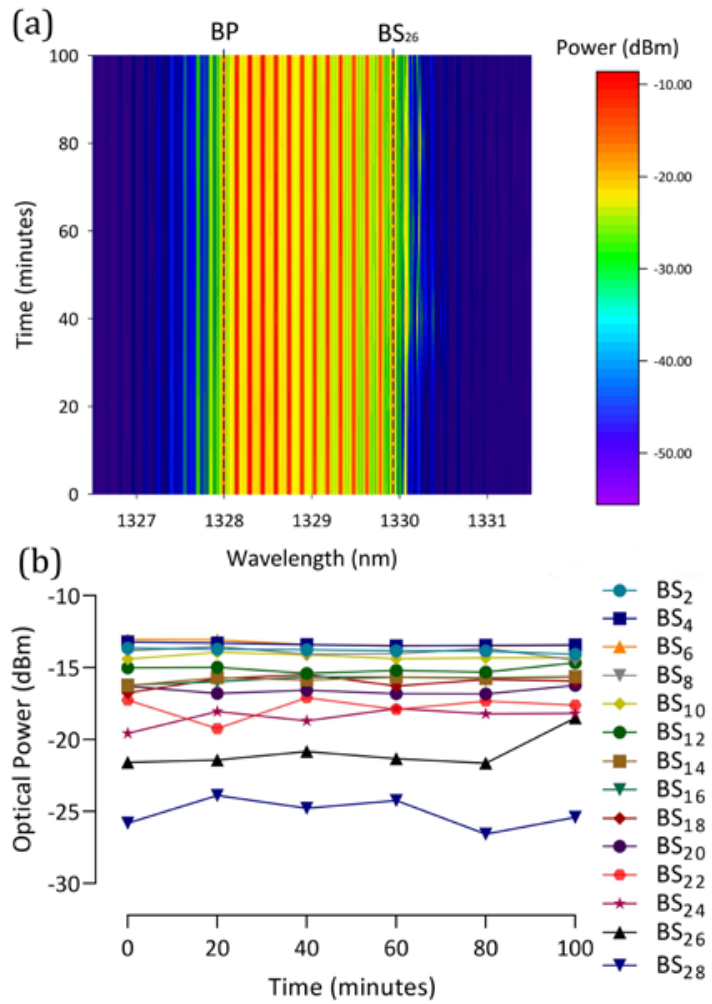

Fig. 9. Stability of the double-spaced MWBBFL in terms of (a) the optical spectrum (top view), and (b) the peak power of each double-spaced Brillouin Stokes lines against time.

Fig. 8 shows the optical spectrum of the MWBBFL with a double frequency spacing taken at the maximum pump power of $1021 \mathrm{~mW}$, where up to 20 Brillouin Stokes and 9 anti-Stokes lines, which were labeled as $2,4,6 \ldots$ were generated. The odd
Stokes are still marginally visible, although they have been greatly attenuated since the position of the output coupler only allow the even Stokes to be extracted from the cavity. The first 13 even Stokes $\left(\mathrm{BS}_{2}\right.$ to $\mathrm{BS}_{26}$ ) have SNRs of between $37 \mathrm{~dB}$ up to $46 \mathrm{~dB}$, while the last 7 even Stokes $\left(\mathrm{BS}_{28}\right.$ to $\left.\mathrm{BS}_{40}\right)$ have SNRs of less than $24 \mathrm{~dB}$. Although the Stokes at longer wavelengths have lower SNRs, the number of Stokes lines generated in this work that have SNRs more than $30 \mathrm{~dB}$ are still higher as compared to that of previously reported works in generating double-spaced Brillouin Stokes [22], [32].
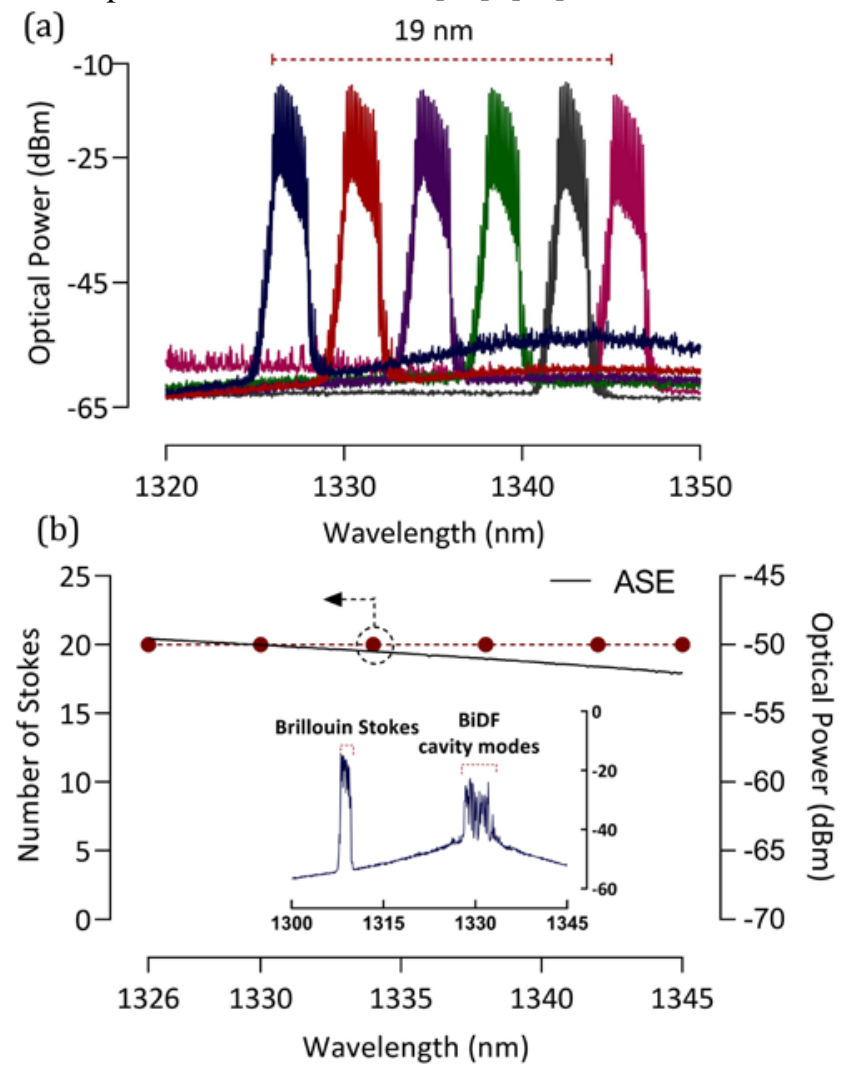

Fig. 10. (a) Tunability spectra of the double-spaced MWBBFL, and (b) the number of generated Brillouin Stokes lines against wavelength. Inset: Appearance of the BiDF cavity modes when the $\mathrm{BP}$ wavelength is tuned beyond the $19 \mathrm{~nm}$ tuning range (1326 $\mathrm{nm}$ to $1345 \mathrm{~nm}$ ).

The stability of the double-spaced MWBBFL is also investigated for a period of 100 minutes. The optical spectrum was taken when the BP wavelength and the pump power was fixed at $1328 \mathrm{~nm}$ and $1021 \mathrm{~mW}$, respectively. The results were recorded at every 20 -minute interval and the stability result is illustrated in Fig. 9 (a). The double-spaced MWBBFL is stable for the first 13 even Stokes $\left(\mathrm{BS}_{2}\right.$ to $\left.\mathrm{BS}_{26}\right)$, in which there is no shift in the wavelengths and no major fluctuations in the peak power of these Stokes lines. The higher order Stokes are less stable, where slight variations in the wavelengths and the peak power could be observed. This could be explained by observing that the lower orders Stokes has actually reached saturation and thus becomes stable. Higher fluctuations were observed for higher order Stokes because the Stokes have not yet reached their saturation levels. This is due to their peak powers being less than $5 \%$ than the peak powers of the stable lower order Stokes. For the peak power analysis of the double-spaced MWBBFL, only even Stokes with peak power of more than $-30 \mathrm{dBm}$ were selected. Thus, only the peak power of the 
TABLE I

DEVELOPMENT OF MWBBFLS IN THE 1.3-MICRON WAVELENGTH REGION.

\begin{tabular}{ccccc}
\hline \hline $\begin{array}{c}\text { Amplifying } \\
\text { medium }\end{array}$ & $\begin{array}{c}\text { Operating } \\
\text { wavelength }(\mathrm{nm})\end{array}$ & $\begin{array}{c}\text { Generated Stokes } \\
\text { (Single / Double) }\end{array}$ & $\begin{array}{c}\text { Tuning Range } \\
(\mathrm{nm})\end{array}$ & Reference \\
\hline SOA & 1310 & $20 /-$ & Fixed & {$[33]$} \\
SOA & 1320 & $11 /-$ & Fixed & {$[34]$} \\
PDFF & $1296-1304$ & $24 / 16$ & $8 / 8$ & {$[23]$} \\
BiDF & $1272-1365$ & $13 / 20$ & $93 / 19$ & [This work] \\
\hline \hline
\end{tabular}

first 14 even Stokes, $\mathrm{BS}_{2}$ to $\mathrm{BS}_{28}$, ranging from $1328.14 \mathrm{~nm}$ to $1330.0741 \mathrm{~nm}$ were recorded in Fig. 9 (b). The minimum and maximum fluctuations of $\mathrm{BS}_{2}$ to $\mathrm{BS}_{16}$ were measured to be only $0.28 \mathrm{~dB}$ and $0.74 \mathrm{~dB}$, respectively, while the remaining Stokes lines, $\mathrm{BS}_{18}$ to $\mathrm{BS}_{28}$, have average fluctuations of about $1.9 \mathrm{~dB}$.

The tunability spectrum of the double-spaced MWBBFL is shown in Fig. 10 (a). The total tuning range for the doublespaced MWBBFL is $19 \mathrm{~nm}$, ranging from $1326 \mathrm{~nm}$ to $1345 \mathrm{~nm}$. A total of 20 even spaced Brillouin Stokes lines were generated at each tuned wavelength, and is the same throughout the whole wavelength span as seen in Fig. 10 (b). This is due to the ASE power being at about the same level from $1326 \mathrm{~nm}$ to $1345 \mathrm{~nm}$, providing enough amplification to only generate 20 Stokes lines. However, the unwanted lasing of the cavity oscillating modes were observed at around the peak of the gain curve as seen in the inset of Fig. 10 (b) when the BP was tuned outside of this $19 \mathrm{~nm}$-wavelength span, causing instability to the generated Brillouin Stokes lines. This phenomenon is common in multiwavelength fiber lasers utilizing the SBS effect [35], [36]. Therefore, we only limit the tuning range of the BP so that the lasing of the BiDF cavity modes was suppressed.

\section{DISCUSSIONS}

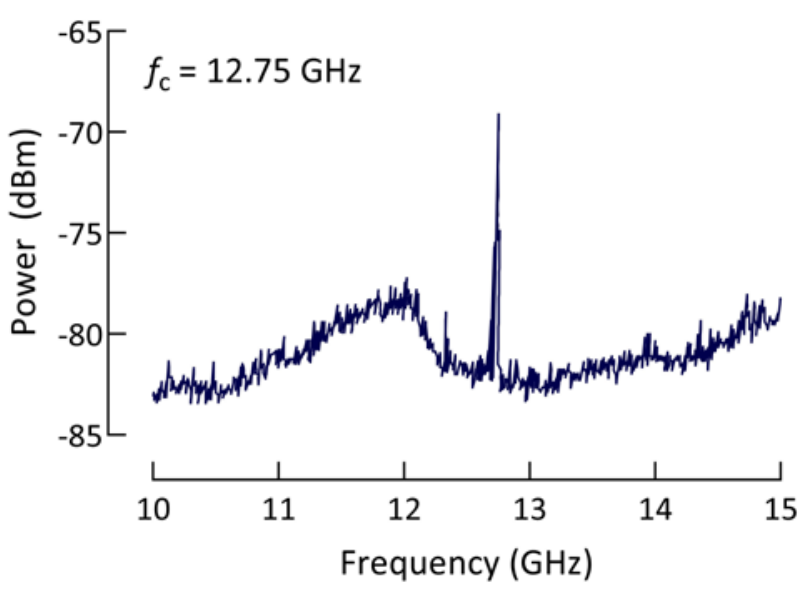

Fig. 11. Frequency beating of the single-spaced MWBBFL

The frequency beating of the MWBBFL was obtained by inputting the output of the laser into a high-speed photodetector (PD). Fig. 11 shows the generated microwave signal after the single-spaced MWBBFL was amplified by an $1.3 \mu \mathrm{m}$ amplifier, and was sent into a Newport 818-BB-35F InGaAs PD. The PD has a bandwidth of $15 \mathrm{GHz}$ and a responsivity is $0.65 \mathrm{AW}^{-1}$ at around $1.31 \mu \mathrm{m}$. The output of the laser needed to be amplified as the frequency response of the PD was relatively low beyond $10 \mathrm{GHz}$. The generated signal has a center frequency of $12.75 \mathrm{GHz}$ as observed in an Anritsu MS2667C Spectrum Analyzer. This is in good agreement with the earlier calculated value of $\sim 12.77 \mathrm{GHz}$. The peak was the result of the superposition of the frequency beatings between the $\mathrm{BP}$ and

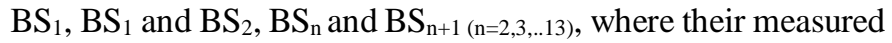
wavelength spacings were identical at about $0.074 \mathrm{~nm}$ $(\sim 12.7 \mathrm{GHz})$. Previous works on multiwavelength fiber lasers based on the SBS effect have also reported similar frequency spacings between adjacent Stokes lines. The previously demonstrated works on multiwavelength Brillouin fiber lasers operating in the $1.3 \mu \mathrm{m}$ wavelength region are given in Table I.

The demand for multi-wavelength Brillouin laser sources operating at $1.3 \mu \mathrm{m}$ is expanding at a significant pace, owing to the increasing demand for a broad bandwidth in DWDM systems. Different types of amplifying medium were explored to provide the necessary gain for the multi-wavelength generation in $1.3 \mu \mathrm{m}$ region, including semiconductor optical amplifiers (SOAs) and praseodymium-doped fluoride fiber (PDFF). Table I compares the performances of multiwavelength Brillouin lasers operating in the $1.3 \mu \mathrm{m}$ region. From the table, it can be seen that the previous works on SOAs are able to provide gain for the generation of single-spacing multi-wavelength Brillouin lasers, centered at $1310 \mathrm{~nm}$ and $1320 \mathrm{~nm}$, respectively. As in [33], 20 Stokes lines were obtained in a ring cavity configuration, while in reference [34] 11 Stokes lines were generated in a linear cavity configuration. The number of generated Stokes lines is nevertheless quite low as compared to that in the PDFF laser cavity, as demonstrated in [23]. Although the number of generated single-spaced Stokes in this work is not as high as that was obtained by Ahmad et al. [23] using PDFF as the gain medium, this would be the first time that Stokes lines are obtained in a cavity using BiDF as the gain medium. This BiDF, unlike the PDFF, can be easily spliced to silica based fibers that are commonplace in optical networks. Furthermore, the BiDF is not hydroscopic as compared to the PDFF which needs extreme care when handling. Additionally, a broader tuning range is observed for both frequency spacings in the BiDF laser cavity as compared to the PDFF, which could be attributed to the broader amplification bandwidth of the BiDF across the $1.3 \mu \mathrm{m}$ region. In this work, the single- and double-spacing multi-wavelength Brillouin lasers can be tuned around $93 \mathrm{~nm}$ and $19 \mathrm{~nm}$, respectively. This is about double the tuning range of the singlespacing Brillouin and about 11 times higher for double-spacing 
Brillouin than that previously reported [23], which makes this laser suitable for various applications especially in DWDM systems.

\section{CONCLUSION}

We have successfully demonstrated the generation of multiwavelength fiber lasers by stimulated Brillouin scattering using bismuth-doped fiber as the laser gain medium. The Brillioun frequency spacing of the multiwavelength laser was switchable between a single- and double-frequency spacing of $\sim 12.75 \mathrm{GHz}$ and $\sim 25.3 \mathrm{GHz}$ respectively. Up to 13 singlespaced and 20 double-spaced Brillouin Stokes lines were generated by placing the BGM in different arrangements in the laser cavity. The MWBBFL can be tuned by varying the central wavelength of the Brillouin pump, where a broad tuning range of $93 \mathrm{~nm}$ was achieved in the single-spaced configuration and up to $19 \mathrm{~nm}$ in the double-spaced configuration respectively. The laser would find potential application in DWDM communication and fiber sensing.

\section{REFERENCES}

[1] J. N. Kemal et al., "Multi-wavelength coherent transmission using an optical frequency comb as a local oscillator," Opt. Express, vol. 24, no. 22, pp. 25432-25445, 2016.

[2] J. J. Veselka and S. K. Korotky, "A multiwavelength source having precise channel spacing for WDM systems," IEEE Photonics Technol. Lett., vol. 10, no. 7, pp. 958-960, 1998.

[3] Y.-G. Han, T. V. A. Tran, S.-H. Kim, and S. B. Lee, "Multiwavelength Raman-fiber-laser-based long-distance remote sensor for simultaneous measurement of strain and temperature," Opt. Lett., vol. 30, no. 11, pp. 1282-1284, 2005.

[4] S. Diaz, D. Leandro, and M. Lopez-Amo, "Stable multiwavelength erbium fiber ring laser with optical feedback for remote sensing," $J$. Light. Technol., vol. 33, no. 12, pp. 2439-2444, 2014.

[5] Z. Wu, Q. Shen, L. Zhan, J. Liu, W. Yuan, and Y. Wang, "Optical Generation of Stable Microwave Signal Using a Dual-Wavelength Brillouin Fiber Laser,” IEEE Photonics Technol. Lett., vol. 22, no. 8, pp. 568-570, 2010.

[6] X. Wang, S. Takahashi, K. Takamasu, and H. Matsumoto, "Space position measurement using long-path heterodyne interferometer with optical frequency comb,” Opt. Express, vol. 20, no. 3, pp. 27252732, 2012.

[7] W. He, W. Zhang, L. Zhu, X. Lou, and M. Dong, "C-band switchable multi-wavelength erbium-doped fiber laser based on Mach--Zehnder interferometer employing seven-core fiber,” Opt. Fiber Technol., vol. 46, pp. 30-35, 2018.

[8] Y. Li et al., "Tunable multiwavelength fiber laser based on a $\theta$-shaped microfiber filter," Appl. Phys. B, vol. 124, no. 6, p. 109, 2018.

[9] L. Zhang, Y. Xu, S. Gao, B. Saxena, L. Chen, and X. Bao, "Multiwavelength coherent Brillouin random fiber laser with ultrahigh optical signal-to-noise ratio,” IEEE J. Sel. Top. Quantum Electron., vol. 24, no. 3, pp. 1-8, 2017.

[10] T. Feng et al., "High stability multiwavelength random erbium-doped fiber laser with a reflecting-filter of six-superimposed fiber-Bragggratings,” OSA Contin., vol. 2, no. 9, pp. 2526-2538, 2019.

[11] Y. Lian, J. Wang, M. Yang, Y. Zhang, and Y. Wang, "Multiwavelength Fiber Laser Using Erbium-Doped Twin-Core Fiber and Nonlinear Optical Loop Mirror,” IEEE Access, vol. 7, pp. 152478-152482, 2019.

[12] S. Yao et al., "Few-mode fiber Bragg grating-based multiwavelength fiber laser with tunable orbital angular momentum beam output," Laser Phys. Lett., vol. 15, no. 9, p. 95001, 2018.

[13] M. Bianchetti et al., "Switchable multi-wavelength laser based on a core-offset Mach-Zehnder interferometer with non-zero dispersionshifted fiber," Opt. Laser Technol., vol. 104, pp. 49-55, 2018.

[14] A. W. Al-Alimi, M. H. A. Bakar, A. F. Abas, M. T. Alresheedi, N. H. Z. Abidin, and M. A. Mahdi, "Stable multiwavelength thulium fiber laser assisted by four wave mixing effect,” Opt. Laser Technol., vol.
106, pp. 191-196, 2018.

[15] G. Mamdoohi, A. R. Sarmani, M. H. A. Bakar, and M. A. Mahdi, "Switchable multiwavelength Brillouin--Raman fiber laser utilizing an enhanced nonlinear amplifying fiber loop design," IEEE Photonics J., vol. 10, no. 2, pp. 1-11, 2018.

[16] J. Galindo-Santos, A. V Velasco, A. Carrasco-Sanz, and P. Corredera, "Brillouin filtering of optical combs for narrow linewidth frequency synthesis,” Opt. Commun., vol. 366, pp. 33-37, 2016.

[17] X. Wang, Y. Yang, M. Liu, and Y. Yao, "Tunable multiwavelength narrow linewidth Brillouin erbium fiber laser based on Rayleigh backscattering,” Opt. Eng., vol. 55, no. 6, p. 66106, 2016.

[18] D. Y. Stepanov and G. J. Cowle, "Properties of Brillouin/erbium fiber lasers,” IEEE J. Sel. Top. quantum Electron., vol. 3, no. 4, pp. 10491057, 1997.

[19] J. Tang, J. Sun, L. Zhao, T. Chen, T. Huang, and Y. Zhou, "Tunable multiwavelength generation based on Brillouin-erbium comb fiber laser assisted by multiple four-wave mixing processes," Opt. Express, 2011.

[20] A. W. Al-Alimi, M. H. Yaacob, A. F. Abas, M. A. Mahdi, M. Mokhtar, and M. H. Al-Mansoori, "150-channel four wave mixing based multiwavelength Brillouin-erbium doped fiber laser," IEEE Photonics J., vol. 5, no. 4, p. 1501010, 2013.

[21] X. Wang, P. Zhou, X. Wang, H. Xiao, and L. Si, "Multiwavelength Brillouin-thulium fiber laser," IEEE Photonics J., vol. 6, no. 1, pp. 17, 2013.

[22] K. Hu, I. V Kabakova, S. Lefrancois, D. D. Hudson, S. He, and B. J. Eggleton, "Hybrid Brillouin/thulium multiwavelength fiber laser with switchable single-and double-Brillouin-frequency spacing," Opt. Express, vol. 22, no. 26, pp. 31884-31892, 2014.

[23] H. Ahmad, S. N. Aidit, and Z. C. Tiu, "Multi-wavelength Praseodymium fiber laser using stimulated Brillouin scattering," Opt. Laser Technol., vol. 99, pp. 52-59, 2018.

[24] R. Gumenyuk, J. Puustinen, A. V Shubin, I. A. Bufetov, E. M. Dianov, and O. G. Okhotnikov, "1.32 $\mu \mathrm{m}$ mode-locked bismuthdoped fiber laser operating in anomalous and normal dispersion regimes," Opt. Lett., vol. 38, no. 20, pp. 4005-4007, 2013.

[25] N. K. Thipparapu, C. Guo, A. A. Umnikov, P. Barua, A. Taranta, and J. K. Sahu, "Bismuth-doped all-fiber mode-locked laser operating at 1340 nm,” Opt. Lett., vol. 42, no. 24, pp. 5102-5105, 2017.

[26] A. Khegai et al., "Bismuth-doped fiber laser at $1.32 \mu \mathrm{m}$ mode-locked by single-walled carbon nanotubes," Opt. Express, vol. 26, no. 18, pp. 23911-23917, 2018.

[27] E. M. Dianov et al., "Bismuth-doped fibre amplifier for the range 1300-1340 nm,” Quantum Electron., vol. 39, no. 12, p. 1099, 2009. G. P. Agrawal, "Stimulated brillouin scattering," in Nonlinear Fiber Optics, 3rd ed. San Diego, CA, USA, Academic Press, 2001, pp. 355384.

[29] P. D. Dragic, "The Acoustic Velocity of Ge-Doped Silica Fibers: A Comparison of Two Models," Int. J. Appl. Glas. Sci., vol. 1, no. 3, pp. 330-337, 2010.

[30] Q. Bao et al., "Monolayer graphene as a saturable absorber in a modelocked laser,” Nano Res., vol. 4, no. 3, pp. 297-307, 2011.

[31] H. Ahmad, N. F. Razak, M. Z. Zulkifli, F. D. Muhammad, Y. Munajat, and S. W. Harun, "Ultra-narrow linewidth single longitudinal mode Brillouin fiber ring laser using highly nonlinear fiber,” Laser Phys. Lett., vol. 10, no. 10, p. 105105, 2013.

[32] Y. G. Shee, A. Ismail, S. Hitam, and M. A. Mahdi, "Multiwavelength Brillouin-erbium fiber laser with double-Brillouin-frequency spacing,” vol. 19, no. 3, pp. 1699-1706, 2011.

[33] A. Hayder and L. R. Chen, "O-band multiwavelength generation in a Brillouin semiconductor fiber laser," in Conference Proceedings Lasers and Electro-Optics Society Annual Meeting-LEOS, 2007.

[34] H. Ahmad, S. F. Norizan, M. Z. Zulkifli, C. H. Pua, Z. A. Ghani, and S. W. Harun, "Investigation of the effects of SOA locations in the linear cavity of an O-band Brillouin SOA fiber laser," J. Mod. Opt., 2011.

[35] B. Dong, D.-P. Zhou, and L. Wei, "Tunable multiwavelength Brillouin-Erbium fiber laser by controlling self-lasing cavity modes' oscillation,” Opt. Fiber Technol., vol. 16, no. 1, pp. 17-19, 2010.

[36] L. Qian, D. Fen, H. Xie, and J. Sun, "A novel tunable multiwavelength Brillouin fiber laser with switchable frequency spacing," Opt. Commun., vol. 340, pp. 74-79, 2015. 


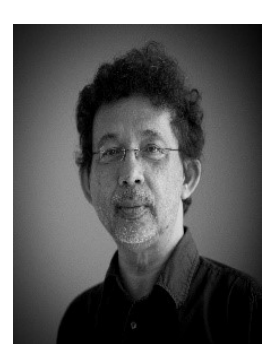

Harith Ahmad received his Ph.D. degree in laser technology from the United of Wales, Swansea, U.K., in 1983. He is currently a Professor with the Department of Physics and the Director of the Photonics Research Centre, University of Malaya, Kuala Lumpur, Malaysia. He has authored over 600 professional papers in international journals and conference proceedings. His research interest includes lasers, pulsed fiber laser, and fiber-based devices for telecommunications.

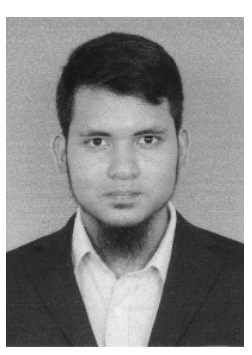

Muhamad Z. Samion obtained his Bachelor of Engineering (Electrical) from the Faculty of Engineering, University of Malaya in 2015. He is currently pursuing his Doctor of Philosophy (PhD) in Photonics Engineering at the Photonics Research Centre, University of Malaya. His research focuses on pulsed and multiwavelength fibre lasers.

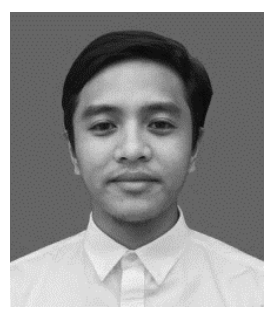

Aizuddin A. Kamely received his B.Sc (Hons) in Pure Physics from International Islamic University Malaysia (IIUM). Currently, he is pursuing his Masters in the field of Photonics Sciences at the Photonics Research Centre, University of Malaya. His research focuses on fiber lasers particularly in the shorter wavelength region of 1.31- and 1.48-micron wavelength region.
Siyi Wang was born in China in 1994. She received her B.Sc. in the field of optoelectronics information technologies at Beihang University, Beijing, China, in 2017. She completed her M.Sc. in Optical Fibre Technologies, from the Optoelectronics Research Centre (ORC), University of Southampton in 2018 and then she continued her study as a Ph.D. in the same field. Her research interests include the development of the Bismuth doped fibre fabrication and the improvement of its laser performance in different wavelength bands.

Yu Wang was born on January 22 ${ }^{\text {nd }}, 1995$ in Zhengzhou, Henan province, China. She received her Bachelor of Science (B.Sc), in field of Optoelectronic Engineering, from the Beihang University, Beijing, China, in 2016. She completed her Master of Science (MSc), in field of Optical Fibre Technologies, from the University of Southampton in 2018 and then joined the Optoelectronics Research Centre, University of Southampton to continue her study of Doctor of Philosophy $(\mathrm{PhD})$ in field of the Development and Application of Broadband Bismuth Doped Fibre Devices.

Jayanta K. Sahu obtained his M.Sc (Physics) and Ph D degrees from the Indian Institute of Technology (IIT) Kharagpur, India. After working at the Royal Institute of Technology, Stockholm, Sweden as a Postdoctoral Research Fellow from 1997 to 2000, he moved to the Optoelectronics Research Centre (ORC), University of Southampton, Southampton, U. K., where he is currently a Professor and is leading the Fibre Fabrication Group. His research focuses on the optical fibre technology, in particular, optical materials, speciality optical fibres, and high-power fibre lasers and amplifiers 